\title{
Standbunkt
}

\section{Von konzeptioneller Willkür und der Glaubwürdigkeit der Wissenschaft}

Der neue Environmental Performance Index 2008, ein weltweites Länder-Ranking der Yale University, misst anhand von 25 Indikatoren, wie ernst es Regierungen mit der Umweltpolitik meinen und welchen Handlungsbedarf sie haben. Eine eigentlich gute Idee, deren Umsetzung aber erheblich zu wünschen übrig lässt. Von Augustin Berghöfer

Ü ber 75 Prozent der deutschen Männer sind zu dick, heißt es im April 2007 in deutschen Tageszeitungen zu einer Studie der Weltgesundheitsorganisation. Die Deutschen sind die Dicksten in Europa! Mit dieser Steilvorlage findet der „Aktionsplan: Fit statt Fett“ der Bundesregierung ein großes Presseecho. Aber kurz darauf zweifeln einige der beteiligten Wissenschaftler an der Aussagekraft der Studie. Unterschiedliche Erhebungszeiträume und Altersgruppen würden Ländervergleiche erschweren (1). Dies zeigt zwei Dinge. Erstens sind Ranglisten ein sehr medienwirksames Instrument, und zweitens sind sie ein methodisch voraussetzungsreiches Konstrukt und damit schwer zu interpretieren. Wenn dies für Übergewicht gilt, dann gilt es sicher auch für Versuche, Umweltpolitik weltweit zu bewerten.

Der Environmental Performance Index (EPI) 2008 ist solch ein Versuch, und er ist grandios gescheitert (2). Auf einer Skala von eins bis 100 bewertet der EPI, inwieweit Länder 25 umweltpolitische Ziele erfüllen. Einzelne Ziele und ihre Indikatoren sind gruppiert in die Politikbereiche Umweltgesundheit, Luft, Wasser, Biodiversität, natürliche Ressourcen und Klima. Indikatorergebnisse werden insgesamt dreimal aggregiert, um so einen gesamten EPI-Wert zu bilden, die Grundlage für das Ranking.

Die Schweiz und skandinavische Länder führen im EPI-Ranking, Costa Rica auf Platz fünf schlägt Deutschland auf Platz 13, und Russland liegt mit Platz 28 deutlich vor den USA auf Platz 39. Nur, was bedeutet das? Es bedeutet weder, dass Ranking-Sieger eine inhaltlich bessere Umweltpolitik machen, noch dass sie mehr Geld, Zeit und Gesetze für Umweltprobleme aufwenden. Der EPI umfasst eine Auswahl von Indikatoren, die sowohl Infrastruktur wie beispielsweise den Zugang zu Trinkwasser, Umweltqualität wie der nationale Holzbestand als auch politische Maßnahmen wie Agrarsubventionen umfassen. Am ehesten beschreibt der EPI also den politischen Handlungsbedarf, das Missverhältnis zwischen Anspruch und Wirklichkeit, aber auch dabei gibt es erhebliche Schwierigkeiten.

\section{Der Index als Messlatte für den Handlungsbedarf}

Nicht alle Ziele sind durch internationale Vereinbarungen gedeckt, und nur fünf der 25 Zielwerte sind durch entsprechende Bestimmungen legitimiert. So wurde der Zielwert, Agrarsubventionen weltweit auf null zu senken, von Experten gesetzt, ohne dafür ein Mandat zu haben. Dies ist nicht nur ein Legitimationsproblem. Macht es Sinn, für alle Länder gleiche Ziele und Zielwerte zu setzen, wenn sie ganz unterschiedliche Probleme haben und Möglichkeiten, diesen zu begegnen?

Ähnlich problematisch ist die Gewichtung. Schwefeldioxid in der Luft fließt mit 1,25 Prozent in den Gesamt-EPI Wert ein, während ein Gesundheitsindikator der Weltgesundheitsorganisation (WHO) 25
Prozent im Gesamt-EPI zählt. Ein Land mit schlechter Gesundheitsversorgung aber kaum Luftverschmutzung, wie zum Beispiel Laos auf Platz 101, schneidet im EPI schlechter ab, als ein Industriestaat mit schlechter Luft und guten Krankenhäusern. Eine gute Begründung dafür fehlt.

\section{Fehlende Daten und unlogische Gewichtung}

Schließlich ist auch die Datenbasis diskussionswürdig. Um alle Länder der Erde abdecken zu können, wird oftmals auf unpräzise Modelldaten zurückgegriffen. Beispielsweise ist in Deutschland laut EPI ein größerer Teil der Landesfläche von Wasserknappheit betroffen als in Mauretanien. Das mögen Modelle so ausrechnen, die nur Entnahme, Niederschlag und Verdunstung berücksichtigen. Das meiste Wasser in Deutschland wird aber zur Kühlung von Kraftwerken gebraucht und flussabwärts wieder eingespeist, während Mauretanien vertrocknet.

In den USA ist der EPI in der Presse verrissen worden. Den Autoren des Index wurde unterstellt, ihn konstruiert zu haben mit dem Ziel, die US-Umweltpolitik zu brandmarken und um Entscheider unter Druck zu setzen. Sicher ist die Umweltpolitik der USA in weiten Teilen unbefriedigend. Aber die konzeptionelle Willkür im EPI ist so offensichtlich, dass die Glaubwürdigkeit der Wissenschaft daran Schaden nimmt (3).

\section{Anmerkungen}

(1) Süddeutsche Zeitung vom 18. April, 9. Mai und 22. Mai 2007

(2) http://epi.yale.edu

(3) Eine ausführliche Untersuchung des EPI findet sich in: Nesshöver, C. / Berghöfer, A. / Beck, S.: Weltranglisten als Beratungsinstrumente für die Umweltpolitik. Marburg 2007.

I AUTOR + KONTAKT

Augustin Berghöfer ist wissenschaftlicher Mitarbeiter am Helmholtz-Zentrum für Umweltforschung (UFZ).

Helmholtz-Zentrum für Umweltforschung $\mathrm{GmbH}$ (UFZ), Permoserstraße 15, 04318 Leipzig. Tel.: +49341 235-1649, E-Mail: augustin.berghoefer@ufz.de, Internet: www.ufz.de 
(c) 20I0 Authors; licensee IÖW and oekom verlag. This is an article distributed under the terms of the Creative Commons Attribution Non-Commercial No Derivates License (http://creativecommons.org/licenses/by-nc-nd/3.o/), which permits unrestricted use, distribution, and reproduction in any medium, provided the original work is properly cited. 